\title{
SUMMARY OF THE STRUCTURE FUNCTION SESSION AT PHOTON 2001*
}

\author{
RICHARD NISIUS \\ CERN, Switzerland, E-mail: Richard.Nisius@cern.ch
}

\begin{abstract}
The status of and ongoing developments in the measurements and theoretical studies of the structure of the photon have been presented at the PHOTON 2001 conference in Ascona. The results presented in the structure function session are briefly summarised.
\end{abstract}

\section{Introduction}

In recent years considerable progress has been made in several aspects of the investigation of the photon structure. A recent review of the status of this field of research can be found int. In this paper, the most important results presented at Ascona are briefly reviewed. Of course the material presented is intended only as an overview, and reflects my personal view. For further information of the important details of the investigations the reader is referred to the write-ups of the individual presentations elsewhere in these proceedings.

\section{Experimental Results}

At this conference new results on the photon structure have been presented by the LEP experiments, using structure functions, and by the H1 and ZEUS experiments, using jet cross-sections. Given the different observables used in electron-photon scattering at LEP and proton-photon scattering at HERA, the variables used in these analyses are different, as illustrated in Figure 1 . The factorisation scale at which the partonic structure of the photon is probed is taken to be $Q^{2}$ in electron-photon scattering, and in proton-photon scattering it is identified with the square of the transverse energy of the jets $E_{\mathrm{T}, \mathrm{jet}}^{2}$. The momentum fraction of the parton in the photon that takes part in the hard interaction is called $x$ in electron-photon scattering, and calculated from $x=Q^{2} /\left(Q^{2}+W^{2}\right)$, where the quantities are shown in Figure 1. In contrast, in di-jet production in proton-photon scattering this quantity is denoted by $x_{\gamma}$ and calculated e.g. from the pseudorapidity $\eta_{\text {jet }}$ and transverse energy of

*INVITED TALK AT THE INTERNATIONAL CONFERENCE ON THE STRUCTURE AND INTERACTIONS OF THE PHOTON, INCLUDING THE 14TH INTERNATIONAL WORKSHOP ON PHOTON-PHOTON COLLISIONS, ASCONA, SWITZERLAND, 2-7 SEPTEMBER, 2001. 

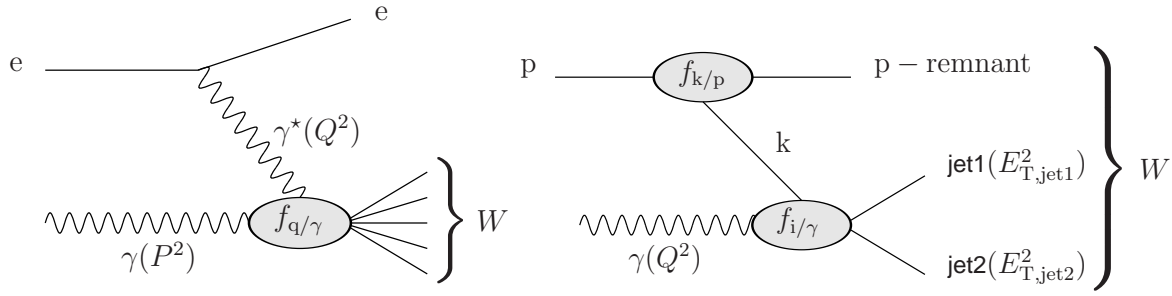

Figure 1. Schematic view of the reactions used in electron-photon (left) and proton-photon scattering (right) for investigations of the photon structure.

the two jets via $x_{\gamma}=\sum_{\mathrm{i}}\left(E_{\mathrm{T}, \text { jeti }} \mathrm{e}^{-\eta_{\text {jeti }}}\right) /(2 y E)$. In addition, some quantities carry different names at LEP and HERA. The virtuality squared of the target photon $\gamma$ in Figure 1, is called $P^{2}$ at LEP and $Q^{2}$ at HERA, and the ratio of the energies of the target photon and the incoming electron, $E_{\gamma} / E$, is called $z$ at LEP and $y$ at HERA.

\subsection{Results from electron-photon scattering}

New results on the hadronic photon structure function $F_{2}^{\gamma}$ have been presented both by DELPHI and by OPALI. The results are based on most of the statistics available at LEP1 and LEP2 energies and extend the measurements of $F_{2}^{\gamma}$ to $\left\langle Q^{2}\right\rangle \approx 750 \mathrm{GeV}^{2}$, see Figure 2 2 for the highest values of $\left\langle Q^{2}\right\rangle$ probed to date.

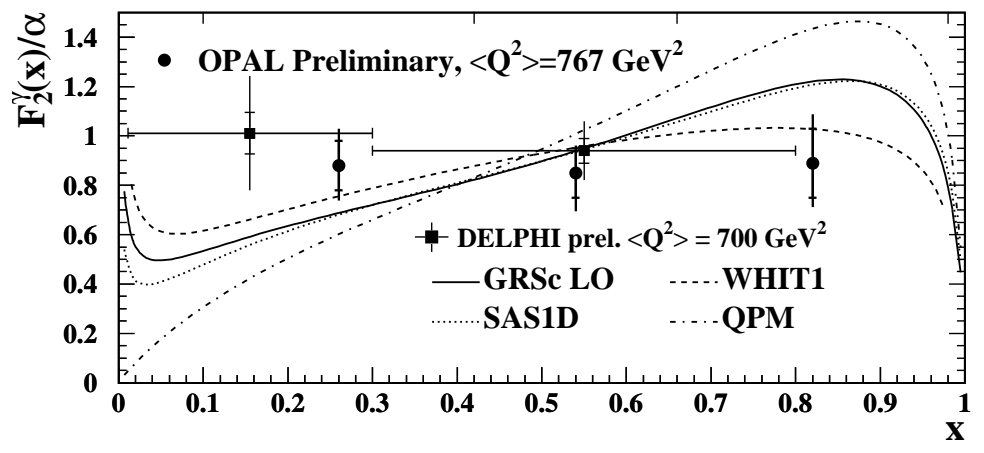

Figure 2. Measurement of the hadronic structure function $F_{2}^{\gamma}$ at the largest $\left\langle Q^{2}\right\rangle$ compared to several parametrisations. 
The strategy to obtain $F_{2}^{\gamma}$ from a measurement of the differential crosssection is still a matter of research. The new DELPHI analysis uses a multivariable fit of observed distributions to adjust the individual components of the hadronic structure of the photon when obtaining $F_{2}^{\gamma}$. This method results in larger uncertainties on $F_{2}^{\gamma}$ than what is achieved by using a one-dimensional unfolding of the distribution of the visible $x$ value of the event, $x_{\mathrm{vis}}$, based e.g. on the RUN program $\theta$, but the claim by DELPHI is that the errors are more reliable for the multivariable fith.

Also the investigation of the evolution of $F_{2}^{\gamma}$ with $Q^{2}$ in ranges of $x$ has been continued using the LEP2 data. As shown in Figure 3, the $Q^{2}$ region has been extended and the errors have been reduced considerably by using the large luminosity available at LEP2 energies. With the present level of

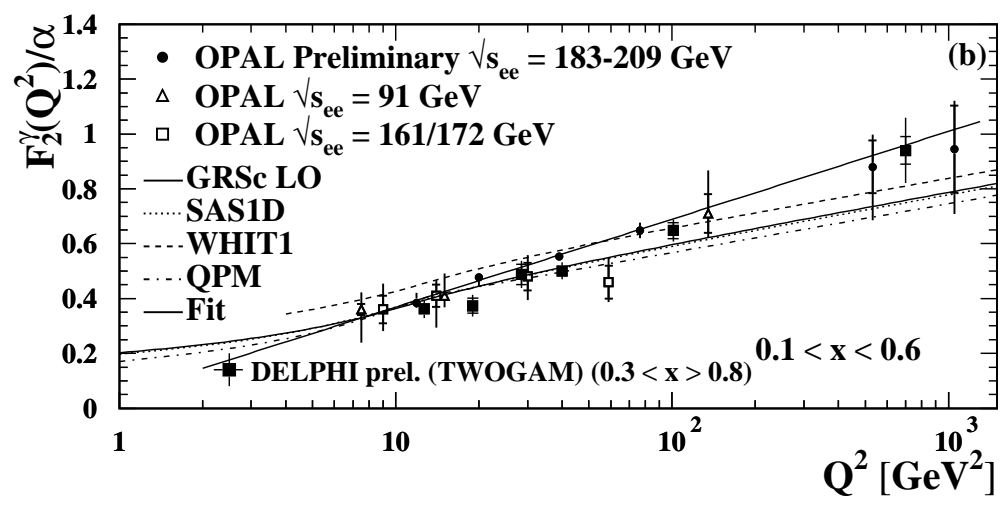

Figure 3. The evolution of $F_{2}^{\gamma}$ with $Q^{2}$ at medium values of $x$ compared to several parametrisations.

statistical precision the data start to challenge the existing parametrisations of $F_{2}^{\gamma}$. Given this, several theoretical as well as experimental issues have to be addressed in more detail. Examples are the suppression of $F_{2}^{\gamma}$ with the virtuality squared of the target photon $P^{2}$ and radiative corrections to the deep-inelastic scattering process.

An update has been presented 0 of the OPAL measurement of the charm component $F_{2, \mathrm{c}}^{\gamma}$ using $D^{\star}$ mesons to identify charm quarks. The analysis is based on improyed Monte Carlo models and higher statistics compared to the published result 9 . This led to an improved precision of the measurement. In a similar way to the structure function for light quarks, $F_{2, \mathrm{c}}^{\gamma}$ receives contributions from the point-like and the hadron-like components of the photon 
structure, as explained e.g. in 1 . These two contributions are predicted to have different dependences on $x$, with the hadron-like component dominating at very low values of $x$ and the point-like part accounting for most of $F_{2, \mathrm{c}}^{\gamma}$ at $x>0.1$. For $x>0.1$ the OPAL measurement is described by perturbative QCD at next-to-leading order. For $x<0.1$ the measurement is poorly described by the NLO prediction using the point-like component alone, and therefore the measurement suggests a non-zero hadron-like component of $F_{2, \mathrm{c}}^{\gamma}$. Increased statistics and a better understanding of the dynamics for $x<0.1$ are needed to get a more precise measurement in this region. To increase the statistics it would be advantageous to combine the data from the four LEP experiments.

\subsection{Results from proton-photon scattering}

New results from H1 andZZES have been presented concerning the structure of quasi-real photons 8 , and also of virtual photons without 9 and with 10 identified charm quarks.

There is good agreement between the ZEUS and H1 results on the inclusive production of jets as can be seen from Figure 1 . The observed inclusive jet cross-sections are well described by existing parton distribution functions of the photon that have been obtained from measurements of $F_{2}^{\gamma}$.

In contrast, there is a longstanding difference between H1 and ZEUS results for di-jet final statesil11. The new preliminary $\mathrm{H} 1$ result 1 , is consistent with the predictions based on existing parametrisations of $F_{2}^{\gamma}$ and at present the data are not precise enough to distinguish between different parametrisations. This has to be confronted with the earlier result from ZEUS12, 8 which suggested that the parton distribution functions of the photon, obtained from fits to measurements of $F_{2}^{\gamma}$ made at $\mathrm{e}^{+} \mathrm{e}^{-}$colliders, are too low for medium values of Bjorken $x$ and at factorisation scales of several hundred $\mathrm{GeV}^{2}$. There are several differences between the ZEUS and H1 analyses such as the choice made for the value of $\alpha_{s}$, the parton distributions used for the photon, and most notably the corrections applied to the data. The H1 data are corrected for detector as well as hadronisation effects and are shown at the partonic level. In contrast, the ZEUS results are corrected only for detector effects and phase space regions are selected, where the hadronisation corrections, as implemented in Monte Carlo models, are found to be small. It remains to be seen how much of the apparent differences between the results can be explained by the different analysis methods. Important information now also comes from LEP, where the parametrisations of $F_{2}^{\gamma}$ are found to be consistent with the measurements for factorisation scales up to $750 \mathrm{GeV}^{2}$, leaving less 


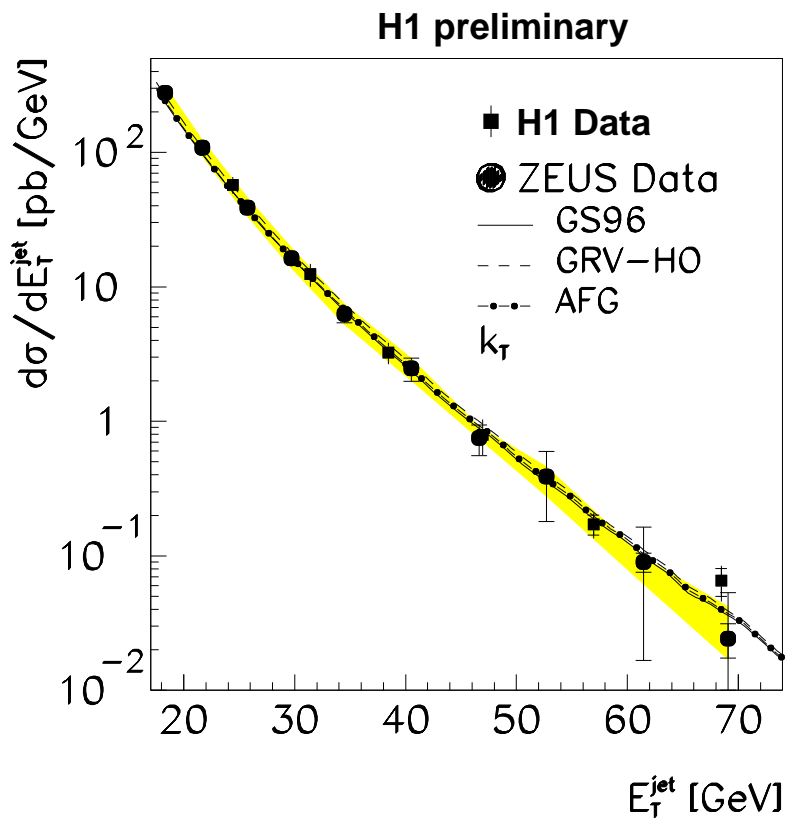

Figure 4. The inclusive jet cross-section as a function of $E_{\mathrm{T}, \text { jet }}$ compared to NLO predictions.

room for changes to the parton distribution functions of the photon.

It is certainly desirable to complement the measurements of $F_{2}^{\gamma}$ with the jet measurements from HERA, which extend to even larger factorisation scales, when fits for the parton distribution functions of the photon are performed. However, first it has to be seen if a consistent picture of the various HERA results can be established.

The measurement of di-jet production has been extended to the investigation of the structure of virtual photons. In the recent ZEUS measurements the suppression of the photon structure with the photon virtuality has been measured based on the ratio of the cross-sections for low and high values of $x_{\gamma}$. The LO predictions fail to describe this ratio when using only transverse virtual photons together with the SaS1D 13 parametrisations of the photon structure. A similar difference has been found by H1. In addition, it has been demonstrated by $\mathrm{H} 1$ that the inclusion of longitudinal virtual photons helps to improve on the description of the observed triple differential cross-section shown in Figure : But the $y$ dependence of the cross-section is still not 


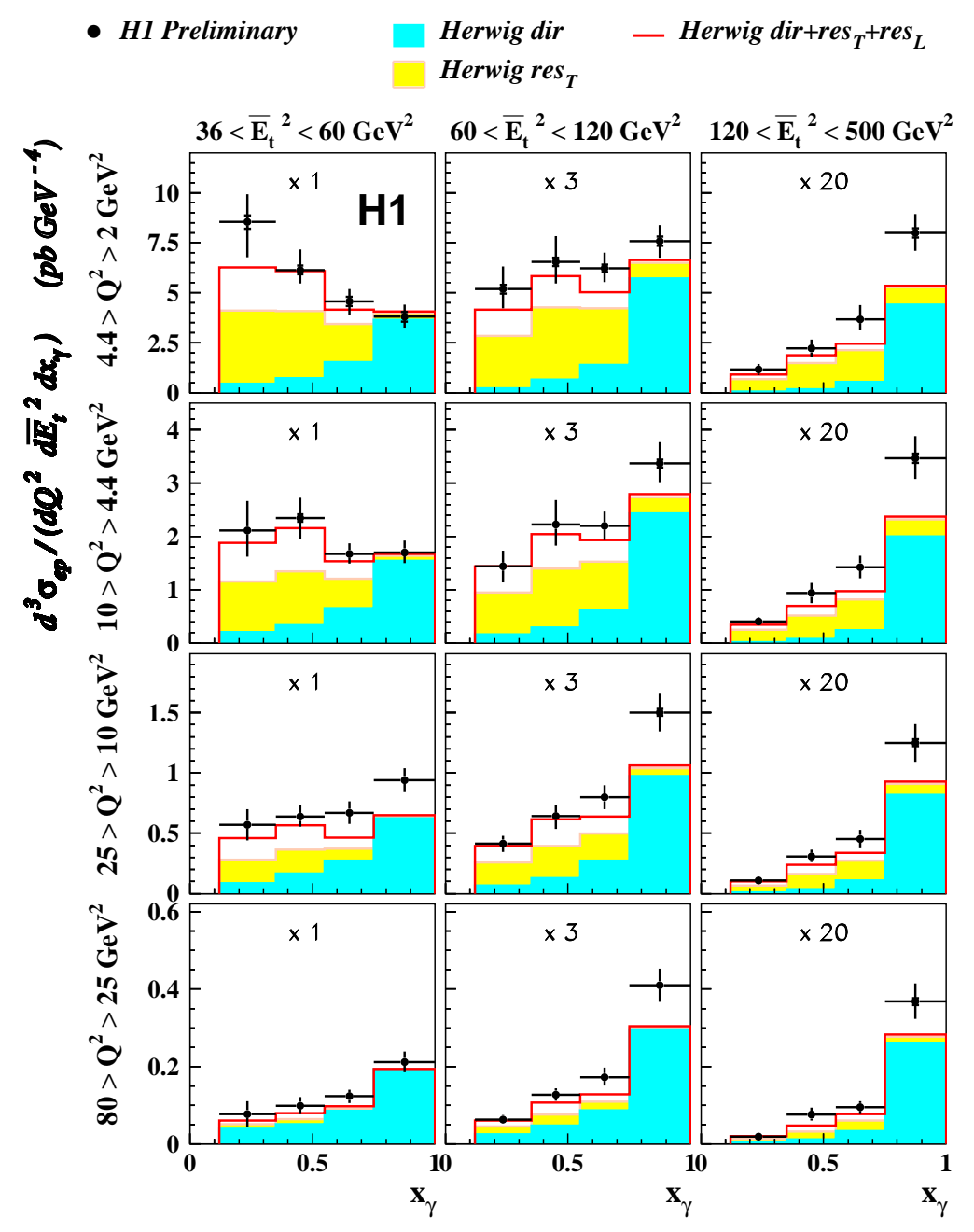

Figure 5. The structure of virtual photons from H1 compared to several predictions from HERWIG.

adequately described for $y<0.3$. More experimental as well as theoretical investigations are needed to better understand these findings.

The structure of virtual photons has also been investigated for the charm component alone, using $D^{\star}$ mesons to identify charm quarks. The mass of 
the charm quark enters as yet another scale in the process, in addition to $E_{\mathrm{T}, \mathrm{jet}}^{2}$ and $Q^{2}$. By again using the ratio of the cross-sections for low and high values of $x_{\gamma}$ it is found that the suppression with $Q^{2}$ is much weaker in the presence of charm than for the sample containing all flavours 10 . The result is less precise than the ratio for all flavours, but, in this case the cross-section ratio is well described by the SaS1D prediction.

\section{Theoretical Developments}

A new parametrisation of $F_{2}^{\gamma}$ has been presented 14 . The extraction of $F_{2}^{\gamma}$ closely follows the procedure used by GRVE 5 in 1992, see alsol, with some changes and additions. The fit uses all modern data on $F_{2}^{\gamma}$. The hadronlike input at the starting scale of the evolution based on VDM is no longer derived from the pion, but fitted to the data, the starting scale of the evolution is varied, heavy quarks are included via the Bethe-Heitler process at all values of $Q^{2}$, and a $\mathrm{J} / \psi$ component is added to the sum of yector mesons. With these ingredients an improved fit to the data is achieved 14 compared to the original GRV parameterisation.

\section{Conclusion}

The investigation of the structure of the photon is a very active field of research experimentally at LEP as well as at HERA, and also theoretically. Given the large statistics available at LEP2 energies and at HERA, the region of phase space covered is constantly increasing. Despite the large luminosities available, for some of the measurements the results are still limited by statistics and a combination of the results from several experiments is desirable. At LEP, this is especially needed for the measurements of $F_{2}^{\gamma}$ at large $Q^{2}$ and for the determination of $F_{2, c}^{\gamma}$. When the differences between the H1 and ZEUS results on di-jet production are resolved, these data will be a valuable input to further constrain the partonic content of the photon.

\section{Acknowledgments}

I like to thank all those who contributed to the structure function session. I greatly appreciate the help that I have been given by the speakers in preparing the summary talk.

\section{References}


1. R. Nisius, Phys. Rep. 332, 165 (2000).

2. I. Tiapkin, these proceedings.

3. R. Taylor, these proceedings, hep-ex/0110024.

4. V. Blobel, Regularized Unfolding for High-Energy Physics Experiments, RUN program manual, unpublished (1996).

5. Á. Csilling, these proceedings.

6. OPAL Collab., G. Abbiendi et al., Eur. Phys. J. C16, 579 (2000).

7. E. Laenen et al., Phys. Rev. D49, 5753 (1994);

E. Laenen and S. Riemersma, Phys. Lett. B376, 169 (1996).

8. A. Valkarova, these proceedings.

9. K. Sedlak, these proceedings.

10. B. West, these proceedings.

11. K. Long, R. Nisius, and W.J. Stirling, Summary of the structure function session at DIS01, hep-ph/0109092.

12. ZEUS Collab., J. Breitweg et al., Eur. Phys. J. C11, 35 (1999).

13. G.A. Schuler and T. Sjöstrand, Z. Phys. C68, 607 (1995).

14. P. Jankowski, these proceedings.

15. M. Glück, E. Reya, and A. Vogt, Phys. Rev. D45, 3986 (1992); M. Glück, E. Reya, and A. Vogt, Phys. Rev. D46, 1973 (1992). 